\title{
Pure spin current from one-photon absorption of linearly polarized light in noncentrosymmetric semiconductors
}

\author{
R.D.R Bhat, F. Nastos, Ali Najmaie, and J.E. Sipe \\ Department of Physics, University of Toronto, 60 St. George Street, Toronto, Ontario, Canada M5S 1 A7
}

(Dated: September 10, 2018)

\begin{abstract}
We show that one-photon absorption of linearly polarized light should produce pure spin currents in noncentrosymmetric semiconductors, including even bulk GaAs. We present $14 \times 14 \mathbf{k} \cdot \mathbf{p}$ model calculations of the effect in GaAs, including strain, and pseudopotential calculations of the effect in wurtzite CdSe.

PACS numbers: 72.25.Fe, 72.25.Dc, 72.40.+w
\end{abstract}

There has been a growing interest in the manipulation of electron spin in semiconductors [1, 2, 3]. Electron spin provides a new degree of freedom to be utilized in novel devices; indeed, several semiconductor 'spintronics' devices have been proposed [4]. Some of these require a spin-polarized electrical current (SPEC) 2]. More exotic is a pure spin current (PSC), in which there is no net motion of charge; spin up electrons travel in one direction while spin down electrons travel in the opposite direction. PSCs could result from spin pumping or Hall effects 5, 6], or interference of two optical beams $[7,8]$. Some of these have been observed [9, 10, 11].

In this Letter, we show theoretically that a ballistic PSC can be generated in noncentrosymmetric semiconductors, merely by linear absorption. This feature of one-photon absorption of linearly polarized light does not seem to have been appreciated previously: It occurs even in unstrained bulk GaAs, it can be generated by a single, weak continuous wave $(\mathrm{CW})$ laser beam, and it arises from the symmetry of the crystal itself.

We consider a semiconductor with filled valence bands and empty conduction bands, uniformly illuminated by light with photon energy larger than the direct band gap. The electric field inside the semiconductor is $\mathbf{E}(t)=$ $\mathbf{E}_{\omega} \exp (-i \omega t)+$ c.c., where $\mathbf{E}_{\omega}$ is a slowly varying envelope function. The coupling between the light and electrons is treated in the long-wavelength limit, and we ignore interactions amongst the electrons. This is a common starting point for calculations of interband absorption in semiconductors [12], and we adopt it to show that PSCs can be generated even within this relatively simple model. We use the semiconductor optical Bloch equations (SOBEs) solved perturbatively to first order in the field intensity [13]. The effect we consider does not rely on carrier scattering, but to connect with experiments, we include an estimate of the spin separation using a simple model of momentum relaxation.

We indicate below that, within these approximations, one-photon absorption of linearly polarized light excites a distribution of electrons in $\mathbf{k}$-space that is even in $\mathbf{k}$, no matter what the symmetry of the material; no net electrical current results 14]. Further, electrons excited into the conduction band at opposite $\mathbf{k}$ points will have oppo- site spin polarization, resulting in no net spin injection. In noncentrosymmetric crystals however, the spin polarization injected at a given $\mathbf{k}$ need not vanish [16]. Thus, in such a case, there will be a PSC, since the velocities of electrons at opposite $\mathbf{k}$ points are opposite.

By treating the field perturbatively, and assuming fast interband dephasing, the SOBE gives for the single particle density matrix $\rho_{n m}(t)$,

$$
\begin{aligned}
\rho_{n m}= & \rho_{n m}^{(\mathrm{ini})}+\sum_{v} \int_{-\infty}^{t} d t^{\prime} \frac{\mathbf{E}_{\omega}\left(t^{\prime}\right) \cdot \mathbf{v}_{n v}}{\omega_{n v}} \frac{\mathbf{E}_{\omega}^{*}\left(t^{\prime}\right) \cdot \mathbf{v}_{v m}}{\omega_{m v}} \\
& \times \frac{\pi e^{2}}{\hbar^{2}} e^{\left(-i \omega_{n m}-\gamma_{n m}\right)\left(t-t^{\prime}\right)}\left(D_{n v}+D_{m v}^{*}\right),
\end{aligned}
$$

where $\rho_{n m}^{(\mathrm{ini})}$ is the initial density matrix, equal to 1 when $n=m$ is a valence band and equal to 0 otherwise, $\omega_{n m}(\mathbf{k}) \equiv \omega_{n}(\mathbf{k})-\omega_{m}(\mathbf{k}), \hbar \omega_{n}(\mathbf{k})$ is the energy of the Bloch state $|n \mathbf{k}\rangle$, the velocity matrix element $\mathbf{v}_{c v}(\mathbf{k}) \equiv\langle c \mathbf{k}|\hat{\mathbf{v}}| v \mathbf{k}\rangle, \gamma_{n m}(\mathbf{k})$ is the dephasing rate, and $D_{n v} \equiv \delta\left(\omega-\omega_{n v}\right)-(i / \pi) \mathcal{P}\left(\omega-\omega_{n v}\right)^{-1}$, where $\mathcal{P}$ indicates the principal part. The dephasing rates approximate many-body interactions [13].

The photoexcited electron density is $N=$ $\sum_{c, \mathbf{k}} \rho_{c c, \mathbf{k}}(t)$. Defining $N_{c v}(\mathbf{k})$ as the electron density excited from band $v$ to band $c$ at $\mathbf{k}$ (i.e. $\left.N=\sum_{\mathbf{k}, c, v} N_{c v}(\mathbf{k})\right)$, we find from Eq. (1) that $\left(d / d t+\gamma_{c c}\right) N_{c v}(\mathbf{k})=\dot{N}_{c v}^{(\mathrm{inj})}(\mathbf{k})$, where

$$
\dot{N}_{c v}^{(\text {inj })}(\mathbf{k}) \equiv \frac{2 \pi e^{2}}{\hbar^{2} \omega^{2}} \frac{1}{V}\left|\mathbf{E}_{\omega} \cdot \mathbf{v}_{c v}(\mathbf{k})\right|^{2} \delta\left(\omega_{c v}(\mathbf{k})-\omega\right),
$$

and $V$ is a normalization volume 12]. Time reversal symmetry of the Bloch states results in Kramers degeneracy, $\omega_{n}(-\mathbf{k})=\omega_{\bar{n}}(\mathbf{k})$, where a bar above a band index denotes the band with the opposite spin. As well, the velocity matrix elements satisfy $\mathbf{v}_{c v}(-\mathbf{k})=-\left[\mathbf{v}_{\bar{c} \bar{v}}(\mathbf{k})\right]^{*}$ 17]. Using these two properties, it follows that when the light is linearly polarized, $\dot{N}_{c v}^{(\mathrm{inj})}(-\mathbf{k})=\dot{N}_{\bar{c} \bar{v}}^{(\mathrm{inj})}(\mathbf{k})$. Consequently, the photocurrent injection rate, given by $e \sum_{\mathbf{k}, c, v}\left[\mathbf{v}_{c c}(\mathbf{k})-\mathbf{v}_{v v}(\mathbf{k})\right] \dot{N}_{c v}^{(\mathrm{inj})}(\mathbf{k})$, is zero for linearly polarized light; hence, any spin current must be a PSC.

The photoexcited electron spin density is $\mathbf{S}=$ $\sum_{c, c^{\prime}, \mathbf{k}}\left\langle c^{\prime} \mathbf{k}|\hat{\mathbf{S}}| c \mathbf{k}\right\rangle \rho_{c c^{\prime}, \mathbf{k}}(t)$, where $\hat{\mathbf{S}}$ is the spin operator. We neglect any spin polarization of the holes, since 
their spin relaxation times are typically very short [18]. Note that for excitation near the band edge $\hbar \omega_{c c^{\prime}}$ is either less than a few $\mathrm{meV}$ (if $c$ and $c^{\prime}$ are equal, or are the spin-split lowest conduction bands) or greater than a few $\mathrm{eV}$ (if $c$ or $c^{\prime}$ is a higher conduction band) 19]. At times $t$ longer than the pulse width $t_{L}$, the integral over $t^{\prime}$ in Eq. (1) will be negligible unless $\omega_{c c^{\prime}}<1 / t_{L}$. For long pulses, a similar argument can be made but with $\gamma_{c c^{\prime}}$ replacing $1 / t_{L}$; however, in this Letter, we focus on typical ultrafast experiments 9, 10], for which one can neglect spin relaxation and carrier recombination occurring on longer timescales. Thus the integral over $t^{\prime}$ allows one to neglect coherences other than those between spin-split pairs of bands. We expand the retained coherences in powers of $\omega_{c c^{\prime}} / \omega$ and keep only the lowest order term. We thus neglect the precession of the spins due to the spin-splitting of the bands; this is justified since the precession period is long compared to the momentum scattering time [3]. Writing $\mathbf{S}=\sum_{\mathbf{k}} \mathbf{S}(\mathbf{k})$, where $\mathbf{S}(\mathbf{k})$ is the electron spin density at $\mathbf{k}$ we find $S^{i}(\mathbf{k})=\sum_{j, l} \zeta^{i j l}(\mathbf{k}) \int_{-\infty}^{t} E_{\omega}^{j *}\left(t^{\prime}\right) E_{\omega}^{l}\left(t^{\prime}\right) d t^{\prime}$, where superscript indices denote Cartesian components,

$$
\begin{aligned}
\zeta^{i j l}(\mathbf{k}) \equiv & \frac{\pi e^{2}}{\hbar^{2} \omega^{2}} \frac{1}{V} \sum_{v, c, c^{\prime}}^{\prime}\left\langle c^{\prime} \mathbf{k}\left|\hat{S}^{i}\right| c \mathbf{k}\right\rangle v_{v c^{\prime}}^{j}(\mathbf{k}) v_{c v}^{l}(\mathbf{k}) \\
& \times\left[\delta\left(\omega_{c v}(\mathbf{k})-\omega\right)+\delta\left(\omega_{c^{\prime} v}(\mathbf{k})-\omega\right)\right],
\end{aligned}
$$

and the prime on the summation indicates a restriction to pairs $\left(c, c^{\prime}\right)$ for which either $c^{\prime}=c$, or $c$ and $c^{\prime}$ are a quasidegenerate pair. Thus the coherence between bands $c$ and $\bar{c}$ is optically excited and grows with the population, as in simpler band models that neglect spin splitting [3]. If one were to neglect the coherence between spin-split bands, the net degree of spin polarization, $|\mathbf{S}| /(N \hbar / 2)$, would only be $10 \%$ in GaAs compared to the accepted $50 \%$ with Eq. (2).

From the time reversal properties of the Bloch states, the spin matrix elements satisfy $\langle n,-\mathbf{k}|\hat{\mathbf{S}}| m,-\mathbf{k}\rangle=$ $-\langle\bar{n}, \mathbf{k}|\hat{\mathbf{S}}| \bar{m}, \mathbf{k}\rangle^{*}$ 17]. Using this property, Kramers degeneracy, and the time reversal property of the velocity matrix elements, one finds $\mathbf{S}(-\mathbf{k})=-\mathbf{S}(\mathbf{k})$ for linear polarized excitation. Thus there is no net spin injection from linearly polarized light, and if $\mathbf{S}(\mathbf{k})$ is nonzero for some $\mathbf{k}$, there is a PSC.

A PSC can be quantified by $\left(I_{\uparrow}-I_{\downarrow}\right)$, where $I_{\uparrow(\downarrow)}$ is the current due to up (down) electrons. A more general measure that naturally accounts for a distribution of carrier velocities and spins is to use a spin current density pseudotensor $K^{i j} \equiv\left\langle\hat{v}^{i} \hat{S}^{j}\right\rangle$ [7].

Phenomenologically, the injection of a spin current due to one-photon absorption can be written in terms of a material response pseudotensor $\mu$, as $K^{i j}=$ $\sum_{l, m} \mu^{i j l m} \int E_{\omega}^{l *}\left(t^{\prime}\right) E_{\omega}^{m}\left(t^{\prime}\right) d t^{\prime}$. The spin current pseudotensor $\mu$ satisfies the intrinsic symmetry $\mu^{i j l m}=$ $\left(\mu^{i j m l}\right)^{*}$. It is further constrained by the symmetry of the material, since it must be invariant under the point group transformations of the crystal. It vanishes for materials with inversion symmetry, but can be nonzero for the symmetries appropriate to zincblende and wurtzite crystals.

The microscopic expression for $\mu$ is derived from Eq. (11), with the same approximations made for Eq. (2). Using time reversal properties of the Bloch functions, one can then show that $\mu$ is real, and can be written as

$$
\begin{aligned}
\mu^{i j l m}= & \frac{\pi e^{2}}{\hbar^{2} \omega^{2}} \frac{1}{V} \sum_{\mathbf{k}, v} \sum_{c, c^{\prime}}^{\prime} \delta\left(\omega_{c v}(\mathbf{k})-\omega\right) \\
& \times \operatorname{Re}\left[\left\langle c^{\prime} \mathbf{k}\left|\hat{v}^{i} \hat{S}^{j}\right| c \mathbf{k}\right\rangle v_{v c^{\prime}}^{l}(\mathbf{k}) v_{c v}^{m}(\mathbf{k})+(l \leftrightarrow m)\right] .
\end{aligned}
$$

In an ultrafast experiment, a PSC can be measured by its displacement of up and down spins. To estimate the spin separation, we use the optically injected electron distribution as a source term in the Boltzmann transport equation. By neglecting space-charge effects, which are negligible for a PSC, the Boltzmann equation can be solved in the relaxation time approximation 10]. This approach neglects scattering during optical excitation, but for ultrafast experiments the error should not be too large. If one measures the spin with respect to the quantization direction $\hat{\mathbf{a}}$, the up and down spin populations are separated by an average displacement $\mathbf{d}(\hat{\mathbf{a}})$. We find $d^{i}(\hat{\mathbf{a}})=\sum_{j}(4 \tau / \hbar) K^{i j} \hat{a}^{j} / N$, where $\tau$ is the momentum relaxation time.

To calculate the PSC for bulk GaAs, we use a $\mathbf{k} \cdot \mathbf{p}$ model that diagonalizes the one-electron Hamiltonian (including spin-orbit coupling) within a basis set of $14 \Gamma$ point states and includes important remote band effects [20]. The 14 states (counting one for each spin) comprise six valence band states (the split-off, heavy, and light hole bands), and eight conduction band states (the two lowest, which are $s$-like, and the six next-lowest, which are $p$-like). The model contains 13 parameters chosen to fit low-temperature experimental data [20].

Strain is included by the deformation potential method of Pikus and Bir 21. The deformation potentials amongst valence and lowest conduction band states are well established [22]. Between the valence and $p$-like conduction band states, there are two deformation potentials for the strain we consider, $a_{c v}$ and $b_{c v}$ 23, 24, 25]. We use $b_{c v}=-2.3 \mathrm{eV}$, which is an average of tight binding calculations [23, 24], and consistent with experiment [26]. The parameter $a_{c v}$ couples to the hydrostatic component of strain 24]; neither it nor the deformation potentials amongst $p$-like upper-conduction band states affect our results. We also neglect the small effect of strain on spinorbit coupling 24].

It is interesting to compare the GaAs results with a material of different symmetry: wurtzite CdSe. Since $\mathbf{k} \cdot \mathbf{p}$ methods are less developed for wurtzite crystals, we 


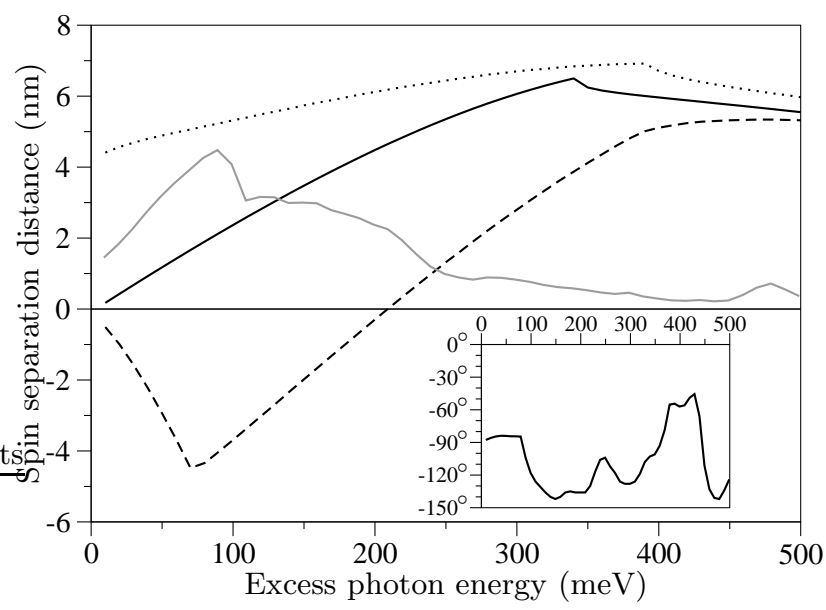

FIG. 1: Calculated spin separation in unstrained GaAs (thick line), in GaAs under 1\% tensile (dotted line) and compressive (dashed line) strain, and in CdSe (grey line). In GaAs, transitions from the split-off band begin to contribute at $\approx 350 \mathrm{meV}$. The inset shows, for CdSe, the angle $\theta$ between $\mathbf{d}(\hat{\mathbf{n}})$ and the $c$ axis; $\theta=45^{\circ}$ corresponds to $\mathbf{d}(\hat{\mathbf{n}}) \| \mathbf{E}_{\omega}$.

use a relativistic pseudopotential plane wave code within the local density approximation [27]; the use of a different method also illustrates the robustness of the PSC effect. Quasiparticle effects are included with a scissor correction. The matrix elements of $\hat{v}^{i} \hat{S}^{j}$ were calculated using $\left\langle c^{\prime} \mathbf{k}\left|\hat{v}^{i} \hat{S}^{j}\right| c \mathbf{k}\right\rangle=\sum_{n} v_{c n}^{i}(\mathbf{k})\left\langle n \mathbf{k}\left|\hat{S}^{j}\right| c \mathbf{k}\right\rangle$ with 16 valence and 24 conduction bands included in the sum; nonlocal contributions to these matrix elements were neglected. The Brillouin zone summation used 2600 irreducible $\mathbf{k}$ points.

For GaAs, we present results for light linearly polarized along [001]. In the standard cubic basis, $K^{x x}=$ $-K^{y y} \equiv \kappa$ are the only two nonzero components of the spin current. Alternately, using a basis where $\hat{\mathbf{x}} \|$ [001], $\hat{\mathbf{z}} \|[110]$ and $\hat{\mathbf{y}} \|[1 \overline{1} 0]$, the only two nonzero components of the spin current are $K^{z y}=K^{y z}=\kappa$. That is, there is a net PSC of electrons with spin component along [11̄0] and velocity component along [110], and an equal PSC with spin component along [110] and velocity component along [110]. Under strain in the [001] direction, the point group symmetry of the crystal is reduced from $T_{d}$ to $D_{2 d}$, but $K^{z y}=K^{y z}$ remain the only nonzero components of the spin current. We have considered $1 \%$ biaxial strain; under tensile (compressive) strain, the lattice dilates (contracts) by $1 \%$ in the [100] and [010] directions and contracts (dilates) by $0.93 \%$ in the [001] direction, with the latter determined from the elastic constants $C_{11}$ and $C_{12}$ 222.

For CdSe, we present results for light linearly polarized at an angle $\pi / 4$ to the $c$-axis. Defining $\hat{\mathbf{n}}$ as a vector perpendicular to both the $c$-axis and the light polarization, we restrict our attention to $\mathbf{d}(\hat{\mathbf{n}})$, which lies in the plane perpendicular to $\hat{\mathbf{n}}$.
The calculated spin separation distances, $|\mathbf{d}|$, for these PSCs are plotted in Fig. 1 as a function of photon energy above the band gap, where we have assumed a momentum relaxation time of $100 \mathrm{fs}$; note that the band gap for each case is different. The inset indicates the direction of the PSC in CdSe. For comparison, experiments on PSCs generated with harmonically related beams in GaAs/AlGaAs quantum wells [9] and ZnSe [10] measured spin separations of $20 \mathrm{~nm}$ and $24 \mathrm{~nm}$ respectively.

To illustrate the spin-momentum correlation implied by the calculation of $\mathbf{d}$, we plot in Fig. 2 calculated angular distributions of the injected carrier density $N(\hat{\mathbf{k}}) \equiv \sum_{|\mathbf{k}|} N(\mathbf{k})$ and degree of spin polarization $\mathbf{s}(\hat{\mathbf{k}}) \equiv\left[\sum_{|\mathbf{k}|} \mathbf{S}(\mathbf{k})\right] / N(\hat{\mathbf{k}})$ for the excited conduction band electrons in GaAs.

In unstrained GaAs, $s_{\max }$, the maximum over $\mathbf{k}$ of $|\mathbf{s}(\hat{\mathbf{k}})| /(\hbar / 2)$, rises from zero at the band edge to a maximum of $12 \%$ at the photon energy when transitions from the split-off valence band become allowed. Under strain, $s_{\max }$ is largest near the band edge. In Fig. 2(b), $s_{\max }$ is $15 \%$ while in Fig. 2(c) it is $44 \%$.

It is clear that strain can increase the PSC, especially at lower energies where strain-induced splitting of the heavy and light hole bands increases the spin polarization of the photogenerated electrons. Under tensile (compressive) strain the light hole band moves to higher (lower) energy than the heavy hole band. The calculated splitting between heavy and light hole bands at the $\Gamma$ point is $84 \mathrm{meV}$ for tensile strain and $69 \mathrm{meV}$ for compressive strain. The crystal field splitting between heavy and light hole bands in CdSe is $38 \mathrm{meV}$.

Note that in Eq. (3) we have assumed that $\hat{\mathbf{v}}$ and $\hat{\mathbf{S}}$ commute. This is no longer true when the anomalous velocity term, $\hbar(\boldsymbol{\sigma} \times \boldsymbol{\nabla V}) /\left(4 m^{2} c^{2}\right)$, is included in $\hat{\mathbf{v}}$. The PSC we have described here does not require that term, in contrast to other effects [6] [28]. In fact, in the $\mathbf{k} \cdot \mathbf{p}$ calculation, the anomalous velocity is neglected. Including $k$-dependent spin-orbit coupling between valence and lowest conduction bands (with $C_{0}=0.16 \mathrm{eV} \AA[29]$ ), and the associated anomalous velocity, changes the calculated spin separation distances by less than $0.1 \mathrm{~nm}$.

While the calculations of ballistic PSCs that we have presented here are appropriate for bulk semiconductors, larger PSCs may be possible in heterostructures, which can be prepared with large structural asymmetry. As well, we note that since only linear absorption is involved, this effect could be studied even with CW beams, and in nanostructures, where short spin transport distances could still have significant consequences.

In a material with low enough symmetry, one-photon absorption of circularly polarized light can generate a ballistic current without an applied voltage [15]; this socalled circular photogalvanic effect (CPGE) is a SPEC [30]. It is tempting to understand the effect we present here as a superposition of opposite SPECs due to two 


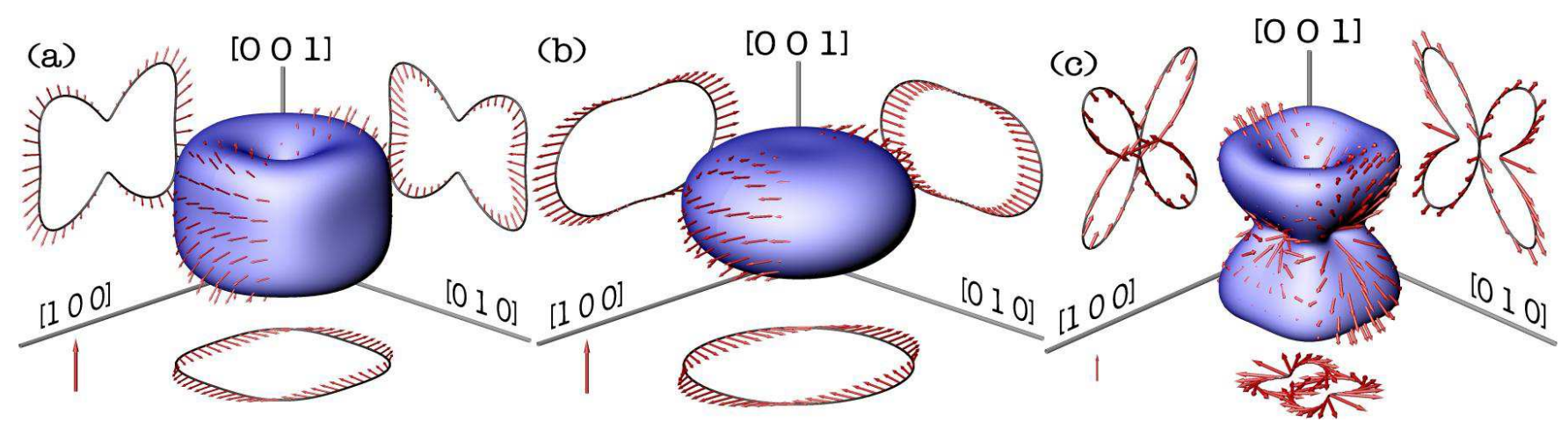

FIG. 2: (Color online) (a) Calculated $\hat{\mathbf{k}}$-space distributions of photoexcited electrons in unstrained GaAs with excess photon energy $300 \mathrm{meV}$. The surface is the injected carrier density, $N(\hat{\mathbf{k}})$, and the vectors affixed to the surface give the degree of spin polarization, $\mathbf{s}(\hat{\mathbf{k}})$, of electrons excited with crystal momentum in the direction $\hat{\mathbf{k}}$. The cross sections through the centers of the distributions, along with the projection of spins on the cross sections, have been displaced to aid visualization. (b) and (c) are as in (a), but at $20 \mathrm{meV}$ excess photon energy and for $1 \%$ tensile and compressive strains respectively. The reference spin in the lower left corner of each panel is $0.25 \hbar / 2$.

fields with opposite helicity. However, the CPGE cannot occur in crystals with zincblende symmetry, although it can in wurtzite CdSe 31] and strained GaAs 32. It is worth noting that in addition to describing the PSC we present here, the spin current response pseudotensor in Eq. (3) can also describe the SPEC due to the the CPGE.

Finally, we point out that a PSC can be generated even with unpolarized light. Averaging over polarization directions in a plane, one obtains $K^{i j}=$ $\int E_{\omega}^{2} d t^{\prime} \sum_{l, m}\left(\delta^{l m}-\hat{n}^{l} \hat{n}^{m}\right) \mu^{i j l m} / 2$, where $\hat{n}$ is the propagation direction of the light. This PSC is smaller than that due to linearly polarized light.

We thank N. Arzate, E. Sherman, Art Smirl and H.M. van Driel for useful discussions. This work was supported by NSERC, PRO and DARPA-SpinS.

[1] S.A. Wolf et al., Science 294, 1488 (2001); D.D. Awschalom and J.M. Kikkawa, Physics Today 52 No. 6, 33 (1999); I. Žutić, J. Fabian. and S. Das Sarma, Rev. Mod. Phys. 76, 323 (2004), and references therein.

[2] Semiconductor spintronics and quantum computation, edited by D.D. Awschalom, D. Loss and N. Samarth (Springer-Verlag, Berlin, 2002).

[3] M.I. Dyakonov and V.I. Perel in Optical Orientation edited by F. Meier and B.P. Zakharchenya, Modern Problems in Condensed Matter Sciences Vol. 8 (NorthHolland, Amsterdam, 1984) Ch. 2.

[4] S. Datta and B. Das, Appl. Phys. Lett. 56, 665 (1990); S. Das Sarma et al., Solid State Commun. 119, 207 (2001), and references therein; J. Rudolph et al., Appl. Phys. Lett. 82, 4516 (2003).

[5] J. E. Hirsch, Phys. Rev. Lett. 83, 1834 (1999); E.R. Mucciolo, C. Chamon, and C.M. Marcus, ibid 89, 146802 (2002); Q.F. Sun, H. Guo and J. Wang, ibid 90, 258301 (2003); W. Long et al., Appl. Phys. Lett. 83, 1397 (2003); T. Aono, Phys. Rev. B 67, 155303 (2003); S. Murakami,
N. Nagaosa, and S.-C. Zhang, Science 301, 1348 (2003); P. Sharma and P.W. Brouwer, Phys. Rev. Lett. 91, 166801 (2003); M. Governale, F. Taddei, and R. Fazio, Phys. Rev. B 68, 155324 (2003); R. Benjamin and C. Benjamin, ibid 69, 85318 (2004); J. Sinova et al., Phys. Rev. Lett. 92, 126603 (2004).

[6] A.G. Mal'shukov, C.S. Tang, C.S. Chu and K.A. Chao, Phys. Rev. B 68, 233307 (2003).

[7] R.D.R. Bhat and J.E. Sipe, Phys. Rev. Lett. 85, 5432 (2000).

[8] A. Najmaie, R.D.R. Bhat and J.E. Sipe, Phys. Rev. B 68, 165348 (2003).

[9] M.J. Stevens et al., Phys. Rev. Lett. 90, 136603 (2003).

[10] J. Hübner et al., Phys. Rev. Lett. 90, 216601 (2003).

[11] S.K. Watson, R.M. Potok, C.M. Marcus, and V. Umansky, Phys. Rev. Lett. 91, 258301 (2003); Y.K. Kato, R.C. Myers, A.C. Gossard, and D.D. Awschalom, Science 306, 1910 (2004); J. Wunderlich, B. Kaestner, J. Sinova, and T. Jungwirth, Phys. Rev. Lett. 94, 047204 (2005).

[12] P.Y. Yu and M. Cardona, Fundamentals of semiconductors (Springer-Verlag, Berlin, 1996) Ch. 6.

[13] H. Haug and S.W. Koch, Quantum theory of the optical and electronic properties of semiconductors (World Scientific, Singapore, 1993); U. Rössler, phys. stat. sol. (b) 234, 385 (2002).

[14] Linear photogalvanic effects, due to asymmetric scattering or a shift in charge density upon excitation can occur 15], but not within these approximations.

[15] B.I. Sturman and V.M. Fridkin, The photovoltaic and photorefractive effects in noncentrosymmetric materials (Gordon and Breach, Philadelphia, 1992).

[16] This was exploited by Alvarado et al., who detected a spin polarization in electrons photoemitted from (110) GaAs; S.F. Alvarado, H. Riechert and N.E. Christensen, Phys. Rev. Lett. 55, 2716 (1985).

[17] The phases of states and partitioning of degenerate subspaces can always be chosen so that this is true; see M. Lax, Symmetry principles in solid state and molecular physics (Dover edition, Mineola, N.Y., 2001).

[18] D.J. Hilton and C.L. Tang, Phys. Rev. Lett. 89, 146601 (2002). 
[19] G. Dresselhaus, Phys. Rev. 100, 580 (1955); M. Cardona, N. E. Christensen, and G. Fasol, Phys. Rev. B 38, 1806 (1988).

[20] P. Pfeffer and W. Zawadzki, Phys. Rev. B 53, 12813 (1996); Of the two parameter sets discussed by Pfeffer and Zawadzki, we use the one corresponding to $\alpha=0.085$ that they find gives better results.

[21] G.E. Pikus and G.L. Bir, Sov. Phys. Solid State 1, 1502 (1959); G.L. Bir and G.E. Pikus, Symmetry and straininduced effects in semiconductors, John Wiley \& Sons (New York, 1974).

[22] I. Vurgaftman, J.R. Meyer and L.R. Ram-Mohan, J. Appl. Phys. 89, 5815 (2001).

[23] B. Koopmans, P.V. Santos, and M. Cardona, phys. stat. sol. (b) 205, 419 (1998).

[24] D. Bertho, J.-M. Jancu and C. Jouanin, Phys. Rev. B 50, 16956 (1994).
[25] A. Blacha, H. Presting, and M. Cardona, phys. stat. sol. (b) 126, 11 (1984).

[26] P. Etchegoin and M. Cardona, Solid State Commun. 82, 655 (1992).

[27] C. Hartwigsen, S. Goedecker and J. Hutter, Phys. Rev. B 58, 3641 (1998); X. Gonze et al., Comput. Mater. Sci. 25, 478 (2002).

[28] E.I. Rashba, Phys. Rev. B 68, 241315 (2003).

[29] T.E. Ostromek, Phys. Rev. B 54, 14467 (1996).

[30] S.D. Ganichev and W. Prettl, J. Phys. Cond. Matter 15, R935 (2003); V.V. Bel'kov et al., Solid State Commun. 128, 283 (2003).

[31] N. Laman et al., Appl. Phys. Lett. 75, 2581 (1999).

[32] Yu.B. Lyanda-Geller and G.E. Pikus, Sov. Phys. Solid State 31, 2068 (1989). 